Acta Crystallographica Section B

Structural

Science

ISSN 0108-7681

Ángel Vegas, ${ }^{a} *$ David

Santamaría-Pérez, ${ }^{a}$ Miriam

Marqués, ${ }^{\mathbf{b}}$ Manuel Flórez,

Valentín García Baonza ${ }^{\mathrm{c}}$ and

J. Manuel Recio ${ }^{\mathrm{b}}$

aInstituto de Química-Física 'Rocasolano', CSIC C/Serrano 119, 28006 Madrid, Spain,

bepartamento de Química Física y Analítica, Universidad de Oviedo, 33006 Oviedo, Spain, and 'Departamento de Química Física, Universidad Complutense de Madrid, 28040 Madrid, Spain

Correspondence e-mail: avegas@iqfr.csic.es

\title{
Anions in metallic matrices model: application to the aluminium crystal chemistry
}

We introduce and discuss an interpretative model of the structure and bonding of inorganic crystals containing metallic elements. The central idea is the conception of the crystal structure of such an inorganic compound as a metallic matrix whose geometric and electronic structures govern the formation and localization of the anions in the lattice. This is the reason for labelling the model anions in metallic matrices (AMM). Taking the $\mathrm{Al} X_{3}$ crystal family $(X=\mathrm{F}, \mathrm{Cl}, \mathrm{OH})$ as a suitable test-bed class of compounds, we illustrate how this approach gives a direct interpretation of the crystalline structures and explains the variable coordination that $\mathrm{Al}$ exhibits in crystalline materials. An exhaustive analysis of the topology of the electron density allows us to provide a quantum-mechanical assessment of the main hypotheses of the AMM model and to uncover, using microscopic arguments, the behavior of anions as chemical pressure agents.

\section{Introduction}

A traditional description of the crystal structures of inorganic compounds consists of an anionic sublattice with interstices partially or totally occupied by cations (Hyde \& Andersson, 1988). Within this view, the local configuration around the individual cations yields the useful concept of (cationic) coordination polyhedra, which has played a key role in the development of crystal chemistry (Hazen \& Finger, 1982). Besides its wide applicability in structural science, this concept constitutes the basis for other interesting applications, namely the transferability of coordination polyhedra compressibilities in equation-of-state models (Hazen \& Finger, 1979) and the visualization of the atomic motions along transition paths in phase transformations (Gracia et al., 2002).

However, in spite of the usefulness and widely recognized importance of the coordination polyhedra approach, the description of crystalline structures within this view meets a major difficulty when attempting to rationalize the coordination number observed for cations in different crystalline environments. The difficulties dramatically increase if the puzzling behavior found in the coordination numbers occurring within the same compound are analysed. Within this approach, it is impossible to predict either the structures accessible to a given material at different pressure and temperature conditions or to explain the underlying reasons for the formation of a particular structure. These shortcomings are clearly manifested in the inability of the approach to provide a general interpretation of the aluminium structures. $\mathrm{Al}$ is a prototypical example because it presents different coordination numbers in different compounds. To name a few:
Received 3 October 2005
(C) 2006 International Union of Crystallography Printed in Great Britain - all rights reserved \author{
Accepted 25 November 2005
}


(i) $\mathrm{Al}$ occupies octahedral voids in $\mathrm{Al}_{2} \mathrm{O}_{3}$ (corundum), $\mathrm{MgAl}_{2} \mathrm{O}_{4}$ (normal spinel), $\mathrm{Al}(\mathrm{OH})_{3}$ and $\mathrm{AlCl}_{3}$, and in the known phases of $\mathrm{AlF}_{3}$;

(ii) it appears tetrahedrally coordinated in many ternary oxides, such as $\mathrm{Ba}_{3} \mathrm{Al}_{2} \mathrm{O}_{6}$ and $\mathrm{LiAlO}_{2}$;

(iii) both types of coordination coexist in other compounds, such as disordered $\mathrm{MgAl}_{2} \mathrm{O}_{4}$ spinels, $\mathrm{Al}_{2} \mathrm{SiO}_{5}$ (sillimanite), $\mathrm{Fe}_{2} \mathrm{Al}_{2} \mathrm{O}_{6}$ and $\mathrm{Sr}_{4} \mathrm{Al}_{14} \mathrm{O}_{25}$;

(iv) $\mathrm{Al}$ appears both penta- and hexa-coordinated in only a few compounds, $\mathrm{Al}_{2} \mathrm{SiO}_{5}$ (andalusite) and $\mathrm{Al}_{6} \mathrm{Ti}_{2} \mathrm{O}_{13}$.

Recently, an alternative approach based on the cation arrangement has been used by Vegas and coworkers to explain part of the above casuistry, i.e. the $\mathrm{Al}$ coordination in aluminates (Santamaría-Pérez \& Vegas, 2003) and $\mathrm{Si}$ in silicates (Santamaría-Pérez et al., 2005). The interpretation is based on both the application of the Zintl-Klemm concept to the cationic arrays and the localization of the anions near the zones of charge accumulation in these cationic arrays. This model is supported by both experimental and theoretical studies on Zintl polyanions and $p$-block elements, which show strong directional covalent bonds and lone pair regions (Coppens et al., 1977; Nesper, 2003).

Empirical evidence has been put forward by O'Keeffe \& Hyde (1985), Borisov and coworkers (Borisov et al., 1998, and references therein), and Vegas and coworkers (Martínez-Cruz et al., 1994; Vegas et al., 2001; Vegas \& Jansen, 2002). First, specific structures of the metal sub-lattices found in different inorganic compounds match those exhibited by the pure metals at different thermodynamic conditions. Among other examples, the structural similarities existing between the $p$ block elements and their corresponding oxides suggest that the structures of oxides are really oxygen-stuffed alloys (Vegas \& Jansen, 2002). Second, the existence of maxima in the distribution of the metal-metal distances along series of compounds at values near the corresponding distances in pure metals points to the existence of some sort of recognition between the metallic atoms. This cationic recognition (CR) hypothesis plays an essential role in determining the crystal structure of compounds (Isea et al., 1998). With respect to the theoretical support, this can be exemplified by the results obtained in ternary aluminates. Application of the ZintlKlemm principle to the cationic sub-arrays results in the donation of electrons from the most electropositive element to $\mathrm{Al}$, giving rise to Zintl polyanions. In these polyanions, the completed octet may be distributed in four pairs of electrons at the vertices of a tetrahedron, the $\mathrm{O}$ atoms located close to these regions of maximum concentration of the electron density, as occurs in $\mathrm{Li}_{5} \mathrm{AlO}_{4}$. Interestingly, in inter-metallic phases containing $\mathrm{Al}$, these regions are also characterized by prominent maxima of the electron localization function (Häussermann et al., 1994), so this formalism seems to be well suited to identify privileged positions in metallic matrices.

However, despite the success of the model to explain the Al coordination in aluminates and silicates, the assumptions must be extended and provided with further support in order to be applied to the interpretation of the structures found in simpler $\mathrm{Al} X_{3}$ compounds $(X=\mathrm{F}, \mathrm{Cl}, \mathrm{OH})$, where the Zintl-Klemm concept is not applicable and the Al sub-lattices do not correspond to any known structure of aluminium. Thus, the aim of this work can be seen as twofold. First, we want to identify and collect the main hypotheses of the model, extending the assumptions to explain the hexa-coordination found for $\mathrm{Al}$ in these binary compounds. Second, we claim to provide a quantum-mechanical verification of all of them, taking the $\mathrm{Al} X_{3}$ crystal family as a reference.

The main advance from our analysis is a far-reaching approach, labeled as anions in metallic matrices (AMM), which provides a direct interpretation of the particular structures shown by crystalline solids containing metallic elements and overcomes some limitations of the previous views (O'Keeffe \& Hyde, 1985; Hyde \& Andersson, 1988). The central idea involves the assumption that the crystal structure of an inorganic compound can be understood as a metallic matrix acting as a host lattice for the non-metallic atoms, the formation and localization of the anions in the compound being driven by the geometric and electronic structures of the metallic sub-lattice. This approach explains the metal-metal connectivity and the metal-non-metal coordination in this type of material and allows the interpretation of the crystalline bonding in terms of the Lewis model. Although the AMM model might be thought to be in agreement with previous observations by O'Keeffe \& Hyde (1985), it introduces new qualitative concepts by taking into account the electronic structure of the metal and chemical bonding models in close affinity with ideas introduced by Nesper (1991), Savin et al. (1997), Vegas et al. (2001) and Vegas \& Jansen (2002). Interestingly, our analysis includes an illustration of the possible equivalence between chemical pressure (oxidation) and thermodynamic pressure proposed by Vegas \& Jansen (2002), as insertion of oxygen into the metallic structure in many cases stabilizes the high-pressure phases of the corresponding metallic matrix (for instance, Si skeletons in silica polymorphs coincide with the known phases of elemental $\mathrm{Si}$ and the tin skeleton in $\mathrm{SnO}_{2}$ resembles the structure of the high-pressure allotrope $\gamma$-Sn).

This paper is organized as follows: $\S 2$ contains the description of the $\mathrm{Al}_{3}(X=\mathrm{F}, \mathrm{Cl}, \mathrm{OH})$ structures. $\S 3$ is divided in two sub-sections. The first sub-section identifies and summarizes the hypotheses of the AMM model emerging from the empirical and theoretical evidence discussed above and from the structural analysis in $\S 2$, and applies them to the $\mathrm{Al} X_{3}$ compounds. The second sub-section provides the theoretical assessment of the AMM hypotheses in terms of:

(i) quantum-mechanical calculations of selected Al sublattices and $\mathrm{Al} X_{3}$ crystals, and

(ii) the analysis of the calculated electron densities by means of the Atoms in Molecules Theory (Bader, 1990; Martín-Pendás \& Luaña, 1995).

Our analysis of the AMM model also includes a microscopic interpretation of the equivalence between chemical pressure and thermodynamic pressure. The paper finishes with a summary of the main conclusions and some prospects of applicability of our model. 


\section{Structural description of the $A \mid X_{3}(X=F, C l, O H)$ compounds}

\section{1. $\mathrm{AlF}_{3}$}

Seven crystalline phases of $\mathrm{AlF}_{3}$ have been reported. In all of them the $\mathrm{Al}$ atom is octahedrally coordinated by $\mathrm{F}$ atoms and the average value of the $\mathrm{Al}-\mathrm{F}$ distances is $1.80 \AA$.

2.1.1. Orthorhombic $\beta$ phase. In the $\beta$ phase of $\mathrm{AlF}_{3}$, Cmcm (LeBail et al., 1988), the Al network consists of 3.6.3.6 planar nets (Kagomé nets) which are connected in the direction perpendicular to the 001 plane with two adjacent Kagomé nets. Each $\mathrm{Al}$ atom has six $\mathrm{Al}-\mathrm{Al}$ contacts: $4 \times 3.465 \AA$ (within the layer) and $2 \times 3.567 \AA$ (between layers). The halogen atoms are located near the centre of the cation-cation contacts, forming slightly distorted octahedra in which each fluorine is common to two adjacent octahedra, forming a corner-connected octahedral network.

2.1.2. Tetragonal phases. Two fluorides have tetragonal symmetry ( $P 4 / \mathrm{nmm}$ and $P 4 / \mathrm{mbm})$. In the former (LeBail et al., 1992), the structure is rather complicated and consists of cavities formed by four puckered pentagonal faces. These cavities are connected by common corners along [110]. The space between these cavities is filled by other cavities, which can approach tetragonal prisms in which their bases are puckered, and by $\mathrm{Al}_{4}$ tetrahedra. The result is that each $\mathrm{Al}$ atom also has six equal $\mathrm{Al}$ neighbours at distances ranging from 3.33 to $3.58 \AA$, located close to the midpoint of the Al$\mathrm{Al}$ contacts (in the case of the largest $\mathrm{Al}-\mathrm{Al}$ contact, the $\mathrm{F}$ atom is located just in the middle of two $\mathrm{Al}$ atoms). The consequence is that each $\mathrm{Al}$ atom is octahedrally coordinated by six $\mathrm{F}$ atoms, all of them being common to two octahedra.

In the latter (Herron et al., 1995), the $\mathrm{Al}$ atoms form planar nets in which triangles, squares and pentagons are present. Within the layer, each $\mathrm{Al}$ atom is four-connected (distances range from 3.44 to $3.579 \AA$ ), but there are two additional Al-
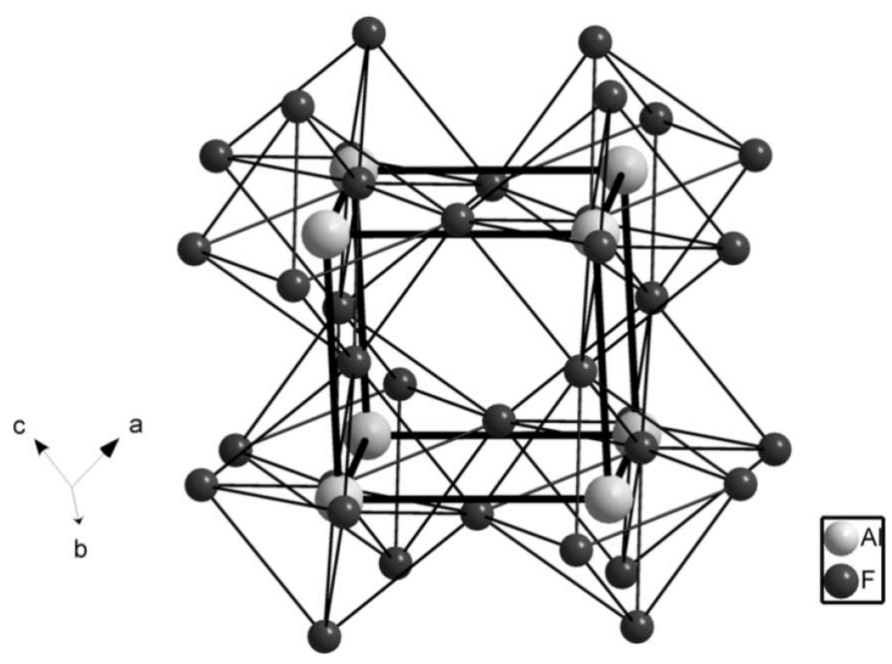

Figure 1

Fragment of the structure of $\alpha-\mathrm{AlF}_{3}$ (rhombohedral). Thick lines connect the $\mathrm{Al}$ atoms to show the similarity with a perovskite structure. Thin lines connect the $\mathrm{F}$ atoms forming the tilted octahedra. As seen, the F atoms lie close to the midpoints of the hypothetical $\mathrm{Al}-\mathrm{Al}$ bonds.
$\mathrm{Al}$ contacts with the two adjacent layers $(2 \times 3.544 \AA)$. As in the other phases, the $\mathrm{Al}$ atoms are six-connected and the $\mathrm{F}$ atoms surrounding the $\mathrm{Al}$ atom are placed near the cationcation contacts giving rise to a corner-sharing $\mathrm{AlF}_{6}$ octahedral framework.

2.1.3. $\mathrm{ReO}_{3}$-type structures. These three phases are rhombohedral, $R 32$ (Ketelaar, 1933), $R \overline{3} H$ (Hoppe \& Kissel, 1984) and $R \overline{3} c$ (Daniel et al., 1990). One of these structures $(R \overline{3} c)$ is represented in Fig. 1. The cationic array is formed by slightly rhombohedrally distorted cubes of $\mathrm{Al}$ atoms with an average $\mathrm{Al}-\mathrm{Al}$ distance of $3.524 \AA$. The $\mathrm{F}$ atoms are placed close to the midpoint of the $\mathrm{Al}-\mathrm{Al}$ contacts, producing an octahedral coordination of the $\mathrm{Al}$ atoms. These tilted $\mathrm{AlF}_{6}$ octahedra share corners forming a skeleton similar to that of $\mathrm{ReO}_{3}$ and to that of orthorhombic perovskites. LeBail et al. (1988) have reported that these rhombohedral phases transform at $725 \mathrm{~K}$ into a cubic phase $(\mathrm{Pm} 3 \mathrm{~m})$ of the $\mathrm{ReO}_{3}$ type, which is really a cubic perovskite with the $A$ cation missing.

2.1.4. Cubic $\eta$-AlF 3 and its relationship with pyrochlore and spinel. A seventh phase of cubic symmetry $(F d \overline{3} m)$ has been reported by Herron et al. (1995) for $\mathrm{AlF}_{3}$. It is obtained by decomposition of the $R^{+} \mathrm{AlF}_{4}^{-}\left(R=\right.$ pyridine $\left.\mathrm{H}^{+}\right)$salt. When the salt is heated, an intermediate product of the formula $\mathrm{HAlF}_{4}$ is obtained. On further heating, the $\eta-\mathrm{AlF}_{3}$ phase is isolated. This phase has been described by Herron et al. (1995) as having a structure identical to pyrochlore materials such as $\mathrm{FeF}_{3}$. It is represented in Fig. 2. In fact, the $\mathrm{Al}^{3+}$ cations only occupy the positions of the $A$ cations in pyrochlore, so this compound could be considered as a variant of the pyrochlore structure in which the $B$ cations are missing. As has been noted by O'Keeffe \& Hyde (1985), the two cation subarrays in

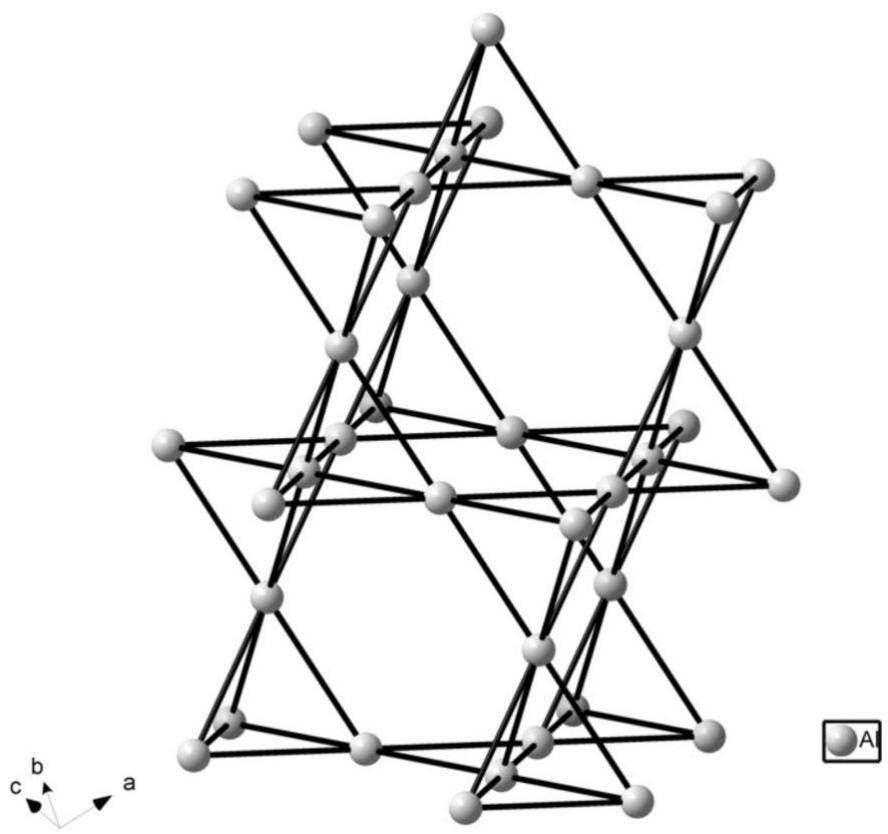

Figure 2

A view of the Al-subarray in cubic $\eta$ - $\mathrm{AlF}_{3}$ showing its similarity with the array of the $B$ cations in pyrochlores and in spinel. The $\mathrm{F}$ atoms would be located close to the midpoint of the $\mathrm{Al}-\mathrm{Al}$ contacts, giving rise to the octahedral coordination. 
pyrochlore are identical to those of the $\mathrm{Al}$ atoms in $\mathrm{MgAl}_{2} \mathrm{O}_{4}$ (spinel) and the $\mathrm{Cu}$ atoms in the Laves phase $\mathrm{MgCu}_{2}$. This net, consisting of a three-dimensional array of $\mathrm{Al}_{4}$ tetrahedra, sharing all their corners, can be seen in Fig. 2, where the sixfold connectivity of the $\mathrm{Al}$ atoms is made evident. As each $\mathrm{F}$ atom is placed close to the line joining two $\mathrm{Al}$ atoms, the result is a three-dimensional network of corner-connected $\mathrm{AlF}_{6}$ octahedra. It is worth mentioning that this framework strongly differs from that observed in spinel where the same $\mathrm{Al}$ subarray, with the $\mathrm{Al}$ atoms also octahedrally coordinated by six $\mathrm{O}$ atoms, gives rise to a three-dimensional network of edge-sharing octahedra. This is due to the different positions adopted by anions $\left(\mathrm{F}^{-}\right.$and $\mathrm{O}^{2-}$, respectively) in both structures. It should be pointed out that the Kagomé nets, constituents of this Al-subarray, are also present in orthorhombic $\beta$ $\mathrm{AlF}_{3}(\mathrm{Cmcm})$. The differences are in the stacking of these 3.6.3.6 nets. In $\beta-\mathrm{AlF}_{3}$, they are stacked in a ...AAA... sequence. When these planar nets are ordered in a ...ABC... sequence, the cubic spinel-like array is formed.

The structural relationship between this cubic phase and the pyrochlore structure has been pointed out by Weber \& Schleid (2000) when discussing the structure of the pyrochlore $\mathrm{Pr}_{2} \mathrm{O}\left(\mathrm{TeO}_{3}\right)_{2}$, whose formulation looks at the pyrochlore structure as a $\mathrm{TeO}_{3}$ network $\left(\mathrm{FeF}_{3}\right.$-type $)$ interpenetrated by another $\operatorname{Pr}_{2} \mathrm{O}$ (anti- $\beta$-cristobalite-type) framework. It should be remembered that a similar description of the pyrochlore structure in terms of this $\mathrm{TeO}_{3}$ network was reported by Wells (1975).

It should be added that the orthorhombic, tetragonal and $\eta$ cubic phases led to $\alpha-\mathrm{AlF}_{3}(R \overline{3} H)$ by heating between 723 and $923 \mathrm{~K}$ (Herron et al., 1995).

\section{2. $\mathrm{AlCl}_{3}$}

In the ICSD (Kirchoff et al., 2004), only one phase of aluminium chloride is reported (Troyanov, 1994). The crystal

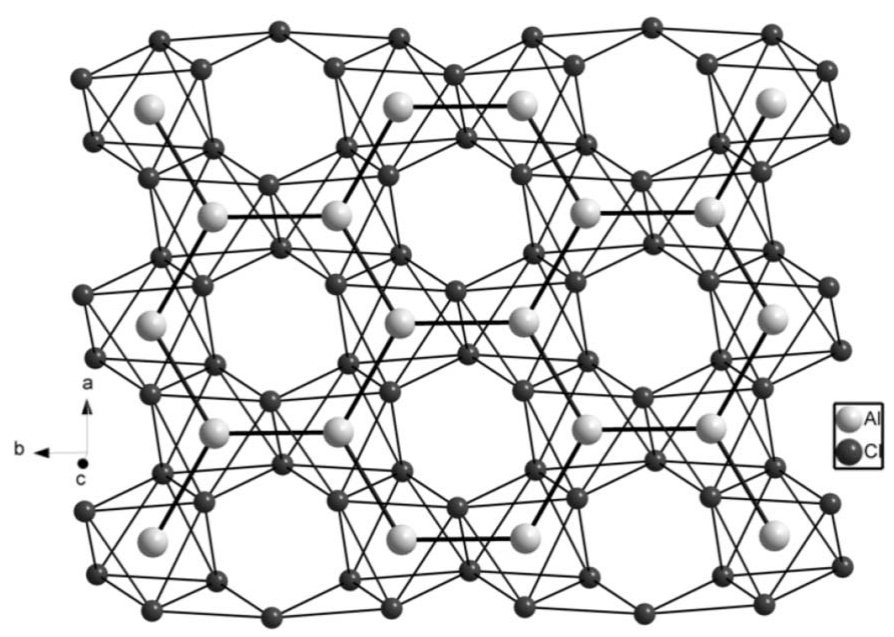

Figure 3

A layer of the $\mathrm{AlCl}_{3}$ structure showing the octahedral coordination of $\mathrm{Al}$ atoms. They are connected by thick lines to show their graphite-like structure. structure is monoclinic $C 2 / m$. The $\mathrm{Al}$ array forms hexagonal graphite-like $\mathbf{6}^{\mathbf{3}}$ layers, packed in an ...AAA... sequence. The hexagons formed are almost regular, with angles close to $120^{\circ}$ and distances ranging from 3.40 to $3.42 \AA$. As in the fluorides, the coordination of the $\mathrm{Al}$ atoms is octahedral, but here the $\mathrm{AlCl}_{6}$ octahedra share edges. One octahedral layer is represented in Fig. 3. This structure is also adopted by the related $\mathrm{InBr}_{3}$ compound.

\section{3. $\mathrm{Al}(\mathrm{OH})_{3}$}

Four phases of aluminium hydroxide have been reported. They correspond to the minerals gibbsite (Saalfeld \& Wedde, 1974), nordstrandite (Bosmans, 1970), bayerite (Zigan et al., 1978) and doyleite (Clark et al., 1998). The structural features are similar to those described previously for $\mathrm{AlCl}_{3}$. In the four phases, the $\mathrm{Al}$ atoms form graphite-like $\mathbf{6}^{\mathbf{3}}$ layers stacked in an ...AAA... sequence. The graphite-like layers are distorted with $\mathrm{Al}-\mathrm{Al}$ distances which range from 2.88 to $2.95 \AA$ and angles varying from 113.5 to $125.8^{\circ}$. This array is similar to that existing in the structure of $\alpha$-Ga (Ramos-Gallardo \& Vegas, 1996). In all of these phases the $\mathrm{Al}$ atoms are octahedrally coordinated by six $\mathrm{OH}$ groups in such a way that each octahedron shares three edges with the three neighbouring octahedra. Fig. 3 can also serve as a reference for these structures.

\section{Anions in the metallic matrices (AMM) model}

\subsection{Hypotheses of the AMM model and application to Al compounds}

Here the hypotheses of the AMM model are collected and applied to interpret the geometry and bonding features of the aluminium binary crystals. The set of hypotheses suggested by the empirical and theoretical evidence discussed in $§ 1$ can be summarized as follows:

(i) the cationic sub-lattices of the inorganic compounds are the reference building blocks of their structures (CR hypothesis);

(ii) in ternary aluminates the Zintl-Klemm concept can be applied to the cationic arrays;

(iii) the simple Lewis electron pair model can be used to associate the metal connectivities with the types and number of non-metallic atoms in the crystals;

(iv) non-metallic elements are located near the zones of charge accumulation in the cationic arrays;

(v) the electron charge density of the metal-metal bonds and lone pairs in the metallic array are transferred to the nonmetallic atoms to form the anions. In addition, the structural features of the $\mathrm{Al} X_{3}$ crystals discussed in $\S 2$ suggest to make explicit a sixth assumption, i.e.

(vi) two types of metal-metal bond may be involved in the charge transference, two-center two-electron bonds and twocenter one-electron bonds.

The AMM model was then applied to interpret the structures of the $\mathrm{Al} X_{3}$ compounds. Firstly, what role does the concept of two-center one-electron bonds play in the interpretation of the hexa-coordination of $\mathrm{Al}$ in $\mathrm{AlF}_{3}$. As shown in 
$\S 2$, Al has a connectivity of six in the cationic arrays of these systems. If each $\mathrm{Al}$ atom provides three valence electrons to the bonding network, hypothetical $\mathrm{Al}-\mathrm{Al}$ bonds would be formed by only one electron (two-center one-electron bonds), close to the midpoint of the $\mathrm{Al}-\mathrm{Al}$ contacts. The AMM model assumes that $\mathrm{F}$ atoms are placed close to these hypothetical $\mathrm{Al}-\mathrm{Al}$ bonds and capture the available electron, thus giving rise to the octahedral coordination of the $\mathrm{Al}$ atoms in the $\mathrm{AlF}_{3}$ crystals. In general, if $\mathrm{Al}$ is connected with six $\mathrm{Al}$ nearest neighbors, then two-center one-electron bonds may be formed and, therefore, a sixfold coordination to mono-negative anions is expected. Secondly, in those Al metal matrices where each $\mathrm{Al}$ is surrounded by three other $\mathrm{Al}$ nearest neighbors, as in $\mathrm{AlCl}_{3}$ or $\mathrm{Al}(\mathrm{OH})_{3}, \mathrm{Al}-\mathrm{Al}$ two-center twoelectron bonds would be formed which represent a naïve way of describing the possibility of either a sixfold coordination to mono-negative anions, as in these two crystals, or a threefold coordination to bi-negative anions. It is, therefore, remarkable that the Lewis model can be used within the AMM context as a useful criterion to distinguish between types of non-metallic atoms and to establish reasonable coordination indexes of the metal in a given compound.

Finally, the role played by the $X$ elements in the final geometry of the $\mathrm{Al} X_{3}$ compounds should be discussed. The non-metallic atom may introduce important volumetric variations on the metallic matrices. The greatest expansion is found when the non-metallic atom acts as a bridge between two metallic atoms, as in two-center one-electron $\mathrm{Al}-\mathrm{Al}$ bonds in $\mathrm{AlF}_{3}$, whereas small modifications may occur in the structures with two-center two-electron $\mathrm{Al}-\mathrm{Al}$ bonds, as in the known phases of $\mathrm{Al}(\mathrm{OH})_{3}$. It is to be pointed out that the shortest $\mathrm{Al}-\mathrm{Al}$ distances observed in these two situations correspond, respectively, to the second and first maxima found in the distribution of $\mathrm{Al}-\mathrm{Al}$ distances in a number of compounds (Isea et al., 1998). Obviously, size effects have to also be considered, as in the case of the $\mathrm{AlCl}_{3}$ crystal, but the overall picture emerging from the above discussion is now satisfactory to account for the observed $\mathrm{Al}-\mathrm{Al}$ distances in $\mathrm{Al} X_{3}$ crystals (see $\S 2$ ).

\subsection{Quantum-mechanical assessment of the AMM model}

The development of the AMM model and the confirmation of the CR and hypotheses (iii)-(vi) require a rigorous detailed study of the electron-density distribution $\rho(x, y, z)$ of the metallic sub-array and its relation to the positions where the anions are located in the crystal. In this sub-section we provide quantum-mechanical support of the AMM approach. The simplest model for metallic crystals is that due to Thomas (1927), Fermi (1927) and Dirac (1930), in which the cationic cores occupy lattice positions and the valence electrons are thrown out to the electron sea generating a quasi-planar surface of charge density. However, a closer analysis of the topology of the electron density in metals reveals slight inhomogeneities in the electron density that can be related to different types of critical points, i.e. non-nuclear maxima, and bond, ring and cage points (Luaña et al., 2003) in the termi- nology of the Atoms in Molecules Theory (AIM) due to Bader (1990). Therefore, our challenge now is to demonstrate that inhomogeneities in the electron density of metals induce favorable sites in the lattice for guest non-metallic atoms. A suitable class of compound is that based on metallic aluminium because the octahedral coordination found in $\mathrm{Al} X_{3}$ compounds $(X=\mathrm{F}, \mathrm{Cl}, \mathrm{OH})$ has been successfully explained in the preceding section in terms of the AMM model and also because, as already indicated above, Al represents a para-
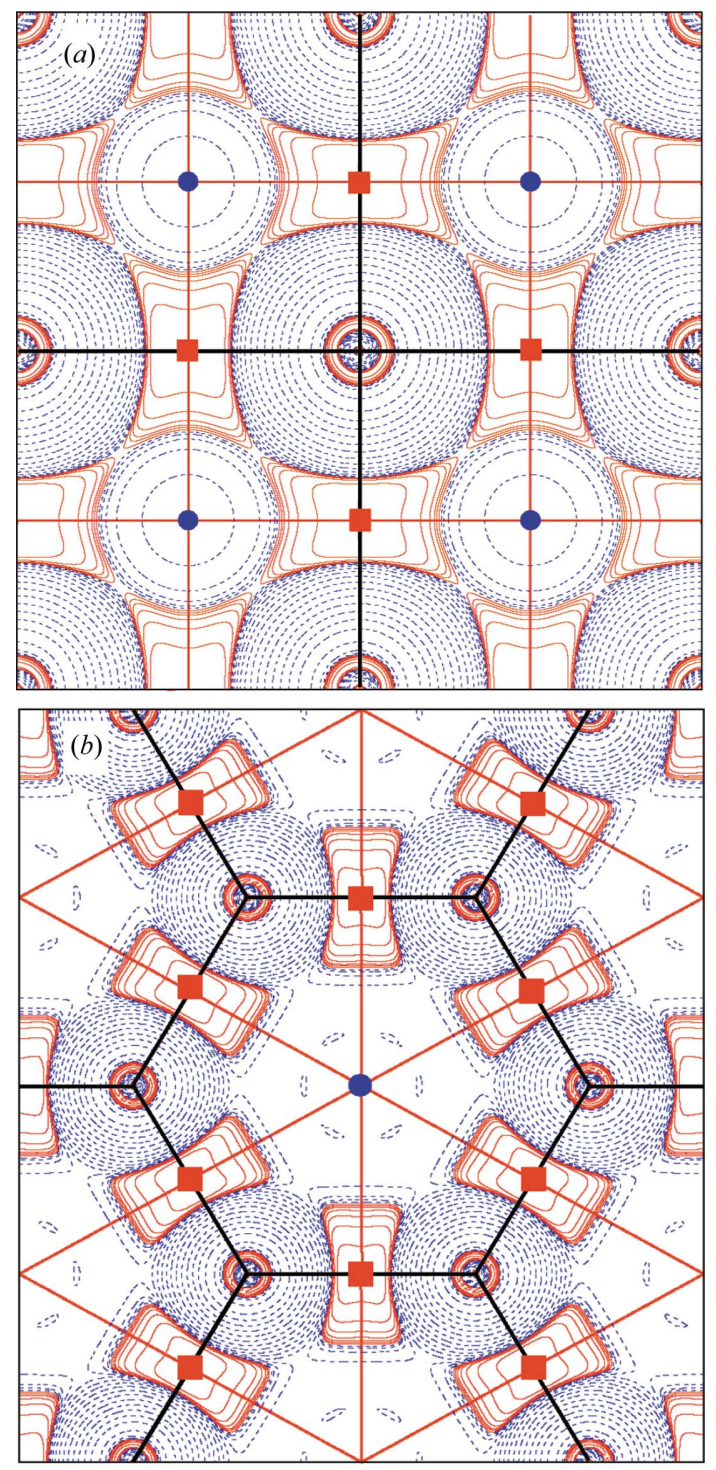

Figure 4

Electron-density Laplacian maps for $\mathrm{Al}$ in $(a)$ the (001) plane of the simple cubic structure and $(b)$ the $(002)$ plane of the idealized eclipsed graphitic structure at their respective equilibrium geometries $(a=2.725$, $a=4.547$, and $c=2.756 \AA$ ). The actual positions of the non-metallic atoms (see Table 1) are dictated by the inhomogenities of the electron density of the host metallic sub-lattice. Inert rare gas atoms tend to be trapped at low-density regions (ring points, blue solid circles), whereas electronegative atoms prefer zones of high density (bond points, red solid squares). Solid (red) and dashed (blue) isolines represent charge depletion and charge accumulation zones, respectively. Bond lines (black solid lines) and atomic boundaries (red solid lines) are also indicated. 


\section{Table 1}

Bond points $(B P)$, cage points $(C P)$ and non-metallic positions in $\mathrm{Al}$ metallic sub-lattices.

All values were calculated in this work, except where indicated. Both $B P$ and $X$ positions correspond to the (common) cells indicated. Hexagonal axes of the $R \overline{3} c$ space group are used. In order to compare the positions of the non-metallic elements in the phases reported for $\mathrm{Al}(\mathrm{OH})_{3}$ and in that reported for $\mathrm{AlCl}{ }_{3}$, the $P 312$ space group has been used for the eclipsed graphitic structure.

\begin{tabular}{llllll}
\hline Structure & $B P$ & $C P$ & $\mathrm{~F}$ & $\mathrm{OH}$ & $\mathrm{Cl}$ \\
\hline$R \overline{3} c$ & $(1 / 2,0,1 / 4)$ & $(0,0,1 / 4)$ & $(0.445,0,1 / 4)$ & $\mathrm{Ne}$ & $(0.356,0,1 / 4)$ \\
& & $(0,428,0,1 / 4)^{(a)}$ & & $(1 / 8,1 / 8,0.426)$ \\
$P d \overline{3} m$ & $(1 / 8,1 / 8,0.511)$ & $(1 / 8,1 / 8,5 / 8)$ & $(1 / 8,1 / 8,0.438)$ & & $(25,0,1 / 4)$ \\
& & $(1,0,1 / 8,0.440)^{(b)}$ & $(2 / 3,0,0.684)^{(c)}$ & $(2 / 3,0.699)^{(e)}$ \\
\end{tabular}

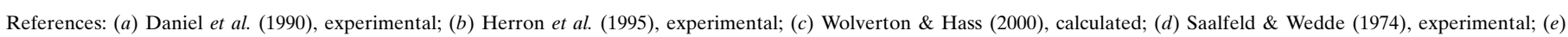
Troyanov (1994), experimental.

digmatic case of bond directionality in metals (Ogata et al., 2002).

We have performed a detailed analysis of the crystalline wavefunctions of metal sub-lattices of selected $\mathrm{Al} X_{3}$ compounds using the Atoms in Molecules formalism, as implemented in the CRITIC code (Martín-Pendás \& Luaña, 1995). Accurate quantum-mechanical calculations have been carried out within the framework of the density functional approximation using standard VASP (Kresse \& Furthmuller, 1996) and CRYSTAL packages (Saunders et al., 1998). Specifically, we have optimized the unit-cell geometries of the following Al lattices: f.c.c. (face-centered cubic; $F m \overline{3} m$ ), b.c.c. (base-centered cubic; $\operatorname{Im} \overline{3} m$ ), h.c.p. (hexagonal-centered cubic; $\left.P 6_{3} / m m c\right)$, simple cubic $(P m \overline{3} m)$, rhombohedral $(R \overline{3})$, spinel $(F d \overline{3} \mathrm{~m})$, eclipsed graphitic $(P 6 / \mathrm{mmm})$, alternated graphitic $(R \overline{3})$ and monoclinic distorted graphite $(C 2 / m)$, as well as the following $\mathrm{Al} X_{3}$ lattices: $\alpha-\mathrm{AlF}_{3}(R \overline{3} c)$ and $\eta-\mathrm{AlF}_{3}$ $(F d \overline{3} m)$, and the hypothetical $\alpha-\mathrm{AlCl}_{3}(R \overline{3} c), \alpha-\mathrm{AlNe}_{3}(R \overline{3} c)$, $\eta-\mathrm{AlCl}_{3}(F d \overline{3} m)$ and $\alpha-\mathrm{AlF}_{3}(P 312)$. As discussed in $\S 2$, the symmetry of the $\mathrm{Al}$ sub-lattice in $\alpha-\mathrm{AlF}_{3}$ and $\eta-\mathrm{AlF}_{3}$ belongs to a slightly distorted simple cubic structure and to a spinel structure, respectively, whereas in various phases of $\mathrm{Al}(\mathrm{OH})_{3}$ and in $\mathrm{AlCl}_{3}$ the $\mathrm{Al}$ sub-lattice can be ideally described with the hexagonal eclipsed graphitic structure (see Figs. 1-3). Complete calculations, including the stability analysis of the Al matrices, and computational parameters are reported in detail elsewhere (Marqués et al., 2006).

The isolines of the Laplacian of the electron density for the simple cubic and hexagonal eclipsed graphitic idealized structures of $\mathrm{Al}$ collected in Fig. 4 contain the basic information sustaining the AMM formalism. The lack of homogeneity of the valence electron density of the metal is clearly revealed by the separation between zones of charge accumulation (negative isolines) and charge depletion (positive isolines). We observe that the bond points (first-order critical points where the electron density is a minimum in one direction, but shows maxima at the other two orthogonal directions) appear just at the center of the $\mathrm{Al}-\mathrm{Al}$ bond lines, inside the regions of negative isolines. This is a very remarkable and general feature also found for all the structures of $\mathrm{Al}$, except the spinel-type lattice where the bond points show a slight departure from the $\mathrm{Al}-\mathrm{Al}$ bond lines. In alkali metals, the same result has also been obtained (Luaña et al., 2003). Interestingly, in all the compounds considered here the nonmetallic atoms $(\mathrm{F}, \mathrm{Cl}, \mathrm{OH})$ are located at positions close to the corresponding bond points of the metallic matrices. In fact, the non-metallic coordinates (see Table 1) lie along the direction connecting the bond points with ring points (where $\rho$ is at a maximum at one direction, but at minima in the two orthogonal directions) or cage points (absolute minima of $\rho$ ), proving that the structure of the metal array and the topology of the electron density are the factors determining the final positions of the anions. Thus, the inhomogeneity of $\rho$ in the metallic lattice around the bond points seems to be replaced by an anion upon crystal formation, in agreement with the electride nature of the metal foreseen by von Schnering \& Nesper (1987). It is also remarkable that the host Al matrices show notably less homogeneous electron density than the pure Al f.c.c. structure (Marqués et al., 2006).

It is interesting now to check whether or not non-metallic atoms with different electronegativities $(\mathrm{F}, \mathrm{Cl}, \mathrm{Ne})$ can accommodate their final positions to the specific details of the Laplacian map of a particular metal matrix. In general, the more (less) electronegative the atom, the closer its position to the bond point (cage point) is expected. Taking the $R \overline{3} c \mathrm{Al}$ sub-lattice as an illustrative example, we optimized the positions of $\mathrm{F}, \mathrm{Cl}$ and $\mathrm{Ne}$ in this structure and found, in agreement with chemical intuition, that the departure of different nonmetallic atoms from the position of the $\mathrm{Al}-\mathrm{Al}$ bond points is modulated with different strength by the presence of the neighboring cage points. In Table 1, a quantitative confirmation to this statement is provided. We find that a continuous decreasing of the $x$ coordinate of $\mathrm{F}(0.445), \mathrm{Cl}(0.356)$ and $\mathrm{Ne}$ (0.25) from the $x$ value at the bond point (0.5) to the $x$ value at the cage point (0.0) correlates with the decreasing electronegativity of these atoms (see the caption to Fig. 4). Similar qualitative results are obtained for the eclipsed graphitic structure (slightly modulated by the fact that two non-metallic atoms approach the bond point). In the spinel lattice, the distance of $\mathrm{Cl}$ to the $B P$ increases with respect to that of $\mathrm{F}$, but the same happens to the corresponding distances to the $C P$ due to the particular positions of the non-metallic atoms in this lattice. It must also be noticed that the $z$ coordinate of the $B P$ in the spinel Al sub-lattice is slightly dependent on the 
volume. Of course, a detailed quantitative explanation of the non-metallic positions in these crystals would require the consideration of the location and nature of all the critical points in the metallic sub-lattice. However, in order to simplify the analysis we have restricted our discussion to the bonding and cage points closer to the positions of the non-metallic atoms. Thus, in lattices having simpler topologies as the $R \overline{3} c$ type, the correlation between non-metallic positions and electronegativities proposed above is more obvious and clearly quantitative, whereas in lattices with more complex topologies, such as the spinel and eclipsed-graphitic structures, this correlation is only qualitative or partially masked. Thus, the actual positions of the non-metallic atoms in crystals result from the compromise between the preference of the guest non-metallic atoms for low electron-density interstices and zones of charge accumulation of the host metal. On general grounds, and under pure energetic considerations, noble-gastype guest atoms find stabilizing van der Waals interactions at the interstices with the lowest electron density, whereas electronegative (non-metallic) atoms tend to occupy the positions of the highest electron density to maximize the Coulomb attractions.

Since we are dealing with the same element, the values of $\rho$ at the BPs in the structures studied are also worth analysis. In those cases where $\mathrm{Al}$ is surrounded by three other $\mathrm{Al}$ atoms, as in the eclipsed graphitic sub-lattice of $\mathrm{Al}(\mathrm{OH})_{3}$, the value of the electron density at the $\mathrm{BP}\left(\rho_{b} \simeq 0.033 \mathrm{e} \mathrm{bohr}^{-3}\right)$ approximately doubles the corresponding value when the connectivity of $\mathrm{Al}$ is six $\left(\rho_{b} \simeq 0.015 \mathrm{e} \mathrm{bohr}^{-3}\right)$, as in the simple cubic and spinel sub-lattices of $\alpha-\mathrm{AlF}_{3}$ and $\eta-\mathrm{AlF}_{3}$, respectively. Although no quantitative correlation should be expected, this result is surprisingly consistent with the simple Lewis picture of the corresponding compounds since two (in the eclipsed graphitic) and one (in the simple cubic and spinel)

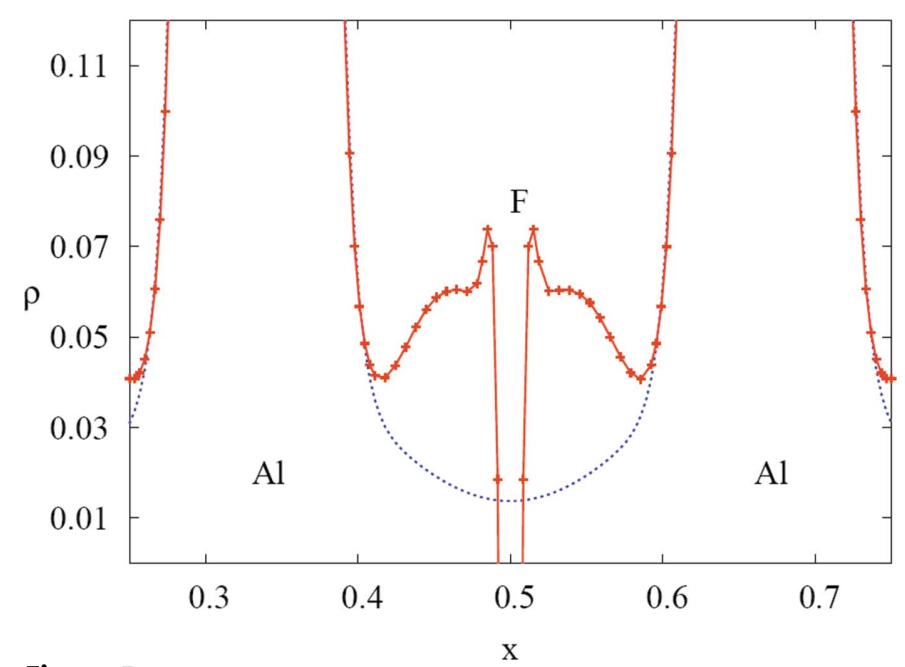

Figure 5

Electron density of $\mathrm{Al}$ atoms along the $\mathrm{Al}-\mathrm{Al}$ bonding line of the $R \overline{3} c$ (slightly distorted simple cubic) structure in two different situations: $(a)$ $\mathrm{Al}$ metal at the experimental geometry of $\alpha-\mathrm{AlF}_{3}$ (dashed line) and (b) $\mathrm{Al}$ metal sub-lattice at the experimental geometry of $\alpha-\mathrm{AlF}_{3}$ after subtraction of the density of neutral $\mathrm{F}$ atoms (continuous line). $\mathrm{Al}$ atoms at $x=1 / 3$ and $x=2 / 3, \mathrm{~F}$ at $x=1 / 2 . \rho$ is given in $\mathrm{e} \mathrm{bohr}^{-3}$ units. electrons are involved in the corresponding $\mathrm{Al}-\mathrm{Al}$ bonds (see \$3.1).

Finally, also relevant to the AMM model is the analysis of the effects of the non-metallic atoms on the electron density of the metal sub-lattice. To this end we have performed a simple computational experiment that consists of plotting the electron density of the pure metal and that of $\mathrm{Al}$ in $\alpha-\mathrm{AlF}_{3}$ along the $\mathrm{Al}-\mathrm{Al}$ bond line of the $R \overline{3} c \mathrm{Al}$ sub-lattice (see Fig. 5). The curve corresponding to $\mathrm{Al}$ in $\alpha-\mathrm{AlF}_{3}$ has been obtained by subtracting the electron density of the neutral $\mathrm{F}$ atoms from the total value of the $\alpha-\mathrm{AlF}_{3}$ crystal. That is, in both curves the electron density originates exclusively from the $\mathrm{Al}$ atoms and, therefore, the volume integration of $\rho$ in the unit cell yields 13 electrons. Although the quantitative use of this curve is not free of ambiguity (numerical inaccuracies appear due to the high values of $\rho$ in the F nucleus and the choice of basis set for neutral $\mathrm{F}$ atoms), the qualitative results are, in our opinion, certainly illuminating.

We observe a clear localization of the electron density towards the $\mathrm{Al}-\mathrm{Al} \mathrm{BP}$, as expected from the high electronegativity of the $\mathrm{F}$ atom located there (strictly speaking, this electron density has to be associated with the final formal charge of $\mathrm{F}$, i.e. with the formation of the $\mathrm{F}^{-}$anion in the ionic $\alpha-\mathrm{AlF}_{3}$ crystal). The $\mathrm{Al}$ electron density in $\alpha-\mathrm{AlF}_{3}$ shown in Fig. 5 might be correlated with that found in alkali metals at reduced unit-cell volumes or elevated hydrostatic pressures (Luaña et al., 2003). This is a very striking aspect of our model that puts forward the well known behavior of anions as chemical pressure agents. Thus, it is expected that there is a reduction of the volume available to the $\mathrm{Al}$ electrons in $\mathrm{Al} X_{3}$ with respect to the volume in the pure $\mathrm{Al}$ metal. In line with the ideas introduced by van der Waals, a non-metallic atom seems to play the role of an additional hard covolume, so its size in the crystal should correlate with the change in pressure induced in the metal sub-lattice. To our knowledge, this is the first microscopic interpretation of this phenomenon.

\section{Conclusions}

We have shown that the geometry and the topology of the electron density of a variety of $\mathrm{Al}$ sub-lattices controls the crystal chemistry (structure and bonding) of aluminium compounds and, in particular, we justified the octahedral coordination of $\mathrm{Al}$ in $\mathrm{Al} X_{3}$ crystals. Our analysis confirms the hypotheses of the AMM model. According to this model, the crystal structure of many inorganic compounds can be understood as resulting from a metallic matrix acting as a host lattice for non-metallic atoms, the electron density of the metal inducing the final positions of the non-metallic atoms in the crystal. The approach is also supported by numerous empirical facts and by quantum-mechanical formalisms of the electron localization function (Nesper, 1991; Savin et al., 1997). The AMM model justifies the observations of O'Keeffe \& Hyde (1985) and explains the relevant role played by the metallic electron density, which differs from the traditional view since our reference is an idealized structure derived from the metallic sub-array of the stable compound. The impor- 
tance of enhancing the role of the metallic matrix might have implications in the description of some solid-state processes where the definition of a meaningful reference state for the non-metallic array is difficult, if not impossible. Our analysis of the AMM model has also put forward the correlation between chemical and thermodynamic pressures and may contribute to understanding the polymorphic sequences induced by pressure and temperature.

This work was supported by the Spanish DGICYT under projects Nos. BQU2001-1695, MAT2004-05867-C03-02 and BQU2003-06553, and Comunidad de Madrid GR/MAT/0358/ 2004. Part of this work was performed under the Project HPCEUROPA (RII3-CT-2003-506079), with the support of the EU Research Infrastructure Action under the FP6 'Structuring the European Research Area' Programme. One of us (DS-P) expresses his thanks to the Residencia de Estudiantes and Ayuntamiento de Madrid for their support.

\section{References}

Bader, R. F. W. (1990). Atoms in Molecules. A Quantum Theory. Oxford University Press.

Borisov, S. V., Podberezskaya, N. V., Pervukhina, N. V. \& Magarill, S. A. (1998). Z. Kristallogr. 213, 253-258.

Bosmans, H. J. (1970). Acta Cryst. B26, 649-652.

Clark, G. R., Rodgers, K. A. \& Henderson, G. S. (1998). Z. Kristallogr. 213, 96-100.

Coppens, P., Yang, Y. W., Blessing, R. H., Cooper, W. F. \& Larsen, F. K. (1977). J. Am. Chem. Soc. 99, 760-766.

Daniel, Ph., Bulou, A., Rousseau, M., Nouet, J., Fourquet, J. L., Leblanc, M. \& Burriel, R. (1990). J. Phys. Condens. Matter, 2, 56635677.

Dirac, P. A. M (1930). Proc. Cambridge Philos. Soc. 26, 376-385.

Fermi, E. (1927). R. Acad. Naz. Lincei, 6, 602-607.

Gracia, L., Beltrán, A., Andrés, J., Franco, R. \& Recio, J. M. (2002). Phys. Rev. B, 66, 224114.

Häussermann, U., Wengert, S., Hofmann, P., Savin, A., Jepsen, O. \& Nesper, R. (1994). Angew. Chem. Int. Ed. Engl. 33, 2069-2073.

Hazen, R. M. \& Finger, L. W. (1979). J. Geophys. Res. 84, 6723-6728.

Hazen, R. M. \& Finger, L. W. (1982). Comparative Crystal Chemistry: Temperature, Pressure, Composition and the Variation of Crystal Structure. New York: John Willey and Sons.

Herron, N., Thorn, D. L., Harlow, R. L., Jones, G. A., Parise, J. B., Fernandez-Boca, J. A. \& Vogt, T. (1995). Chem. Mater. 7, 75-83.

Hoppe, R. \& Kissel, D. (1984). J. Fluorine Chem. 24, 327-340.
Hyde, B. G. \& Andersson, S. (1988). Inorganic Crystal Structures. New York: John Wiley and Sons.

Isea, R., Vegas, A. \& Ramos-Gallardo, A. (1998). Acta Cryst. B54, 35 40.

Ketelaar, J. A. A. (1933). Z. Kristallogr. Abt. A, 85, 119-131.

Kirchoff, A., Pebler, A., Warkentin, E., Bergerhoff, G. \& Luksch, P. (2004). FIZ, Karlsruhe, ICSD.

Kresse, G. \& Furthmuller, J. (1996). Phys. Rev. B, 54, 11169-11186.

LeBail, A., Fourquet, J. L. \& Bentrup, U. (1992). J. Solid State Chem. 100, 151-159.

LeBail, A., Jacoboni, C., LeBlanc, M., De-Pape, R., Duroy, H. \& Fourquet, J. L. (1988). J. Solid State Chem. 77, 96-101.

Luaña, V., Mori-Sánchez, P., Costales, A., Blanco, M. A. \& MartínPendás, A. (2003). J. Chem. Phys. 119, 6341-6350.

Marqués, M., Flórez, M., Recio, J. M., Santamaría-Pérez, D. \& Vegas, A. (2006). Submitted for publication.

Martín-Pendás, A. \& Luaña, V. (1995). The CRITIC code is available from the authors upon request (angel@fluor.quimica.uniovi.es).

Martínez-Cruz, L. A., Ramos-Gallardo, A. \& Vegas A. (1994). J. Solid State Chem. 110, 397-398.

Nesper, R. (1991). Angew. Chem. Int. Ed. Engl. 30, 789-817.

Nesper, R. (2003). Silicon Chemistry, edited by P. Jutzi \& U. Schubert. Weinheim: Wiley-VCH.

Ogata, S., Li, J. \& Yip, S. (2002). Science, 298, 807-811.

O'Keeffe, M. \& Hyde, B. G. (1985). Structure and Bonding, Vol. 61, pp. 77-144. Berlin: Springer Verlag.

Ramos-Gallardo, A. \& Vegas, A. (1996). Z. Kristallogr. 211, 299-303.

Saalfeld, H. \& Wedde, M. (1974). Z. Kristallogr. 139, 129-135.

Santamaría-Pérez, D. \& Vegas, A. (2003). Acta Cryst. B59, 305-323.

Santamaría-Pérez, D., Vegas, A. \& Liebau, F. (2005). Structure and Bonding, Vol. 118. Berlin: Springer Verlag.

Saunders, V. R., Dovesi, R., Roetti, C., Causa, M., Harrison, N. M., Orlando, R. \& Zicovich-Wilson, C. M. (1998). CRYSTAL98 User's Manual. University of Torino, Italy.

Savin, A., Nesper, R., Wengert, S. \& Fässler, T. F. (1997). Angew. Chem. Int. Ed. Engl. 36, 1809-1832.

Schnering, H. G. von \& Nesper, R. (1987). Angew. Chem. Int. Ed. Engl. 26, 1059-1080.

Thomas, L. H. (1927). Proc. Cambridge Philos. Soc. 23, 542-548.

Troyanov, S. I. (1994). Zh. Neorg. Khim. 39, 552-555.

Vegas, A., Grzechnik, A., Syassen, K., Loa, I., Hanfland, M. \& Jansen, M. (2001). Acta Cryst. B57, 151-156.

Vegas, A. \& Jansen, M. (2002). Acta Cryst. B58, 38-51.

Weber, F. A. \& Schleid, T. (2000). Z. Anorg. Allg. Chem. 626, 12851287.

Wells, A. F. (1975). Structural Inorganic Chemistry. Oxford: Clarendon Press.

Wolverton, C. \& Hass, K. C. (2000). Phys. Rev. B, 63, 024102.

Zigan, F., Joswig, W. \& Burger, N. (1978). Z. Kristallogr. 148, 255-273. 